\section{The pain of integrins}

Although chronic pain is a common problem, progress in treating it has been relatively slow. Advances in our understanding of neuropathic and inflammatory pain are important if new therapeutic targets are to be identified. In the European Journal of Neuroscience, Dina and colleagues describe a potential role for integrins in mediating this kind of pain.

Integrins are adhesion molecules that are expressed on primary afferent neurons. They bind to elements of the extracellular matrix (ECM) and interact with signalling systems that are known to mediate hyperalgesia. Inflammation and nerve injury cause changes in the ECM around peripheral nerves that might provide important signals to integrins. So, the authors investigated whether integrins were involved in the sensitization of nociceptors that leads to hyperalgesia in animal models of inflammatory and neuropathic pain.

Dina et al. used several methods to interfere with normal integrin signalling in rats, and found that by doing so they could block the development of hyperalgesia. However, there seems to be more than one pathway through which integrins can influence pain.

Fragments of laminin, or antibodies against the integrin subunits that are involved in binding to laminin, blocked hyperalgesia induced by injection of prostaglandin $\mathrm{E}_{2}$ $\left(\mathrm{PGE}_{2}\right)$, but not that induced by injection of adrenaline (epinephrine).

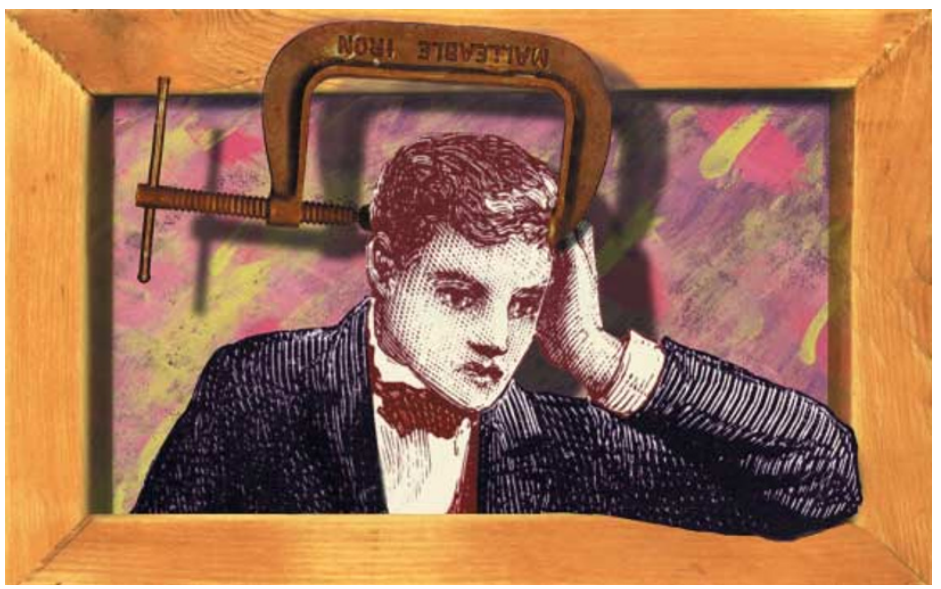

The laminin peptides also blocked the induction of hyperalgesia in a sloweracting model of inflammatory pain, the injection of carrageenan. On the other hand, fragments of fibronectin, or antibodies against the subunits of integrin that are needed for bindng fibronectin, prevented the induction of hyperalgesia by adrenaline, but not by $\mathrm{PGE}_{2}$ or by carrageenan.

The $\beta_{1}$ integrin subunit is involved in the binding of both laminin and fibronectin. When rats were treated with antibodies against this subunit, neither adrenaline nor $\mathrm{PGE}_{2}$ induced hyperalgesia. The same effect was obtained by treatment with antisense oligodeoxynucleotides that knocked down the expression of the $\beta_{1}$ subunit. The knockdown also prevented hyperalgesia in a model of neuropathic pain caused by the chemotherapeutic drug taxol, and could reverse hyperalgesia if given after taxol treatment.

This evidence that specific integrin subunits are involved in pathways that cause pain when stimulated by different substances should add to our growing understanding of the mechanisms of chronic pain. It might also lead to the development of new therapies for the many patients who are affected by inflammatory or neuropathic pain.

Rachel Jones

(2) References and links ORIGINAL RESEARCH PAPER Dina, O. A. et al. Integrin signaling in inflammatory and neuropathic pain in the rat. Eur. J. Neurosci. 19, 634-642 (2004)

\section{GENE EXPRESSION}

\section{Stochastic yet biased expression of multiple Dscam splice variants by individual cells.}
Neves, G. et al. Nature Genet. 1 February 2004 (10.1038/ng1299)
The Drosophila homologue of Dscam is essential for axon guidance and undergoes alternative splicing to generate 38,016 possible isoforms. Neves et al. used single-cell RT-PCR to investigate the expression of Dscam in single photoreceptors. The authors find that individual photoreceptors express 14-50 mRNAs from the possible range of Dscam isoforms, and that populations of photoreceptor subtypes express broad but distinctive subsets of Dscam. They propose that the Dscam repertoire of a cell could provide a mechanism for generating unique cell identity in the nervous system. 